Oper Orthop Traumatol 2013 · 25:4-4

DOI 10.1007/s00064-013-0232-9

๑) Springer-Verlag Berlin Heidelberg 2013

Schriftleitung Operative Orthopädie und Traumatologie

\title{
Die Zeitschrift Operative Orthopädie und Traumatologie dankt den Gutachtern 2012
}

Prof. Dr. Christoph Eingartner

Klinik für Orthopädie und

Unfallchirurgie, Caritas-

Krankgenhaus Bad Mergentheim

PD Dr. Norman Espinosa

Fuß- \& Sprunggelenkschirurgie,

Uniklinik Balgrist, Zürich, Schweiz

Prof. Dr. Karl-Heinz Frosch

Chirurgisch-Traumatologisches Zentrum,

Asklepios Klinik St. Georg, Hamburg

Dr. Thomas Fuchs

Klinik und Poliklinik für Unfall-, Handund Wiederherstellungschirurgie, Universitätsklinikum Münster

Prof. Dr. Emanuel Gautier

Klinik für Orthopädische

Chirurgie und Traumatologie,

Kantonsspital Fribourg, Schweiz

Prof. Dr. Florian Gebhard

Klinik für Unfall-, Hand-, Plastische und Wiederherstellungschirurgie, Universitätsklinikum Ulm

Prof. Dr. Rolf Haaker

Orthopädie und Traumatologie,

St. Vincenz-Hospital, Brakel

Dr. Horst Haferkamp

Kassel

Dr. Uta Hahn

Klinik für Orthopädie und

Orthopädische Chirurgie, Krankenhaus

Dresden-Friedrichstadt

Prof. Dr. Johannes Hamel

Orthopädie Zentrum Arabellapark

(OZA), München

Prof. Dr. Robert Hierner

Department für Plastische,

Rekonstruktive, Ästhetische

und Handchirurgie, Bethanien-

Krankenhaus Moers

PD Dr. Maik Hoberg

Orthopädische Klinik, König-

Ludwig-Haus Würzburg

Prof. Dr. Marcel Jakob Universitätsspital Basel, Schweiz

PD Dr. Chlodwig Kirchhoff

Klinik und Poliklinik für Unfallchirurgie, Klinikum rechts der Isar, Technische Universität München
Prof. Dr. Martin Krismer

Universitätsklinik für Orthopädie,

Medizinische Universität Innsbruck

Prof. Dr. Andreas Kurth

Orthopädische Klinik und Poliklinik,

Universitätsmedizin Mainz

Dr. Stefan Lakemeier

Abteilung Orthopädie,

Universitätsmedizin Göttingen

Prof. Dr. Wolfgang Linhart

Klinik für Unfallchirurgie und

Orthopädie, Handchirurgie,

SLK-Kliniken Heilbronn

PD Dr. Thomas Mückley

Klinik für Unfallchirurgie, Orthopädie und Fußchirurgie, Klinikum Coburg

Prof. Dr. Lars Müller

Klinik und Poliklinik für

Orthopädie und Unfallchirurgie,

Universitätsklinikum Köln

Prof. Dr. Luca Papavero

Klinik für Spinale Chirurgie,

Schön Klinik Hamburg Eilbek

Dr. Thomas Pillukat

Klinik für Handchirurgie der Herz- und Gefäß-Klinik GmbH, Bad Neustadt/Saale

Dr. Michael Plecko

Klinik für Unfallchirurgie,

UniversitätsSpital Zürich, Schweiz

PD Dr. Matthias Rab

Plastische, Ästhetische und

Rekonstruktive Chirurgie, Klinikum

Klagenfurt am Wörthersee, Österreich

Dr. Jörg Rether

Klinik für Unfall- und

Wiederherstellungschirurgie,

Berufsgenossenschaftliche

Unfallklinik Tübingen

PD Dr. Andreas Roth

Klinik für Orthopädie und

Unfallchirurgie, Waldkrankenhaus

"Rudolf Elle", Eisenberg

PD Dr. Stephan Sehmisch Abteilung für Unfallchirurgie, Plastische und Wiederherstellungschirurgie, Universitätsmedizin Göttingen

Prof. Dr. Klaus Siebenrock Universitätsklinik für Orthopädische Chirurgie und Traumatologie, Inselspital, Universitätsspital Bern, Schweiz
PD Dr. Christoph Siepe

Wirbelsäulenchirurgie, Schön Klinik München Harlaching

\section{Dr. Richard Stange}

Klinik und Poliklinik für Unfall-, Handund Wiederherstellungschirurgie, Universitätsklinikum Münster

Prof. Dr. Hajo Thermann Zentrum für Hüft-, Knie- und Fußchirurgie, Sporttraumatologie, ATOS Klinik Heidelberg

PD Dr. Frank Unglaub Orthopädie, Orthopädische Chirurgie und Unfallchirurgie, Vulpius Klinik Bad Rappenau

Dr. Hans-Joachim Walde Klinik für Unfallchirurgie und Orthopädie, Nordwest-Krankenhaus Sanderbusch, Sande

Prof. Dr. Markus Walther Fußchirurgie Sprunggelenkchirurgie, Schön Klinik München Harlaching

Prof. Dr. Johannes Zeichen Klinik für Unfallchirurgie und Orthopädie, Johannes Wesling Klinikum Minden
Dr. Oliver Eberhardt

Orthopädische Klinik, Klinikum Stuttgart 